\title{
Irregularity Measures of Subdivision Vertex-Edge Join of Graphs
}

\author{
Jialin Zheng, ${ }^{1}$ Shehnaz Akhter, ${ }^{2}$ Zahid Iqbal, ${ }^{2}$ Muhammad Kashif Shafiq, ${ }^{3}$ \\ Adnan Aslam $\mathbb{D}^{4},{ }^{4}$ Muhammad Ishaq, ${ }^{2}$ and Muhammad Aamir $\mathbb{D D}^{5}$ \\ ${ }^{1}$ School of Information Science and Technology, Chengdu University, Chengdu 610106, China \\ ${ }^{2}$ School of Natural Sciences, National University of Sciences and Technology, H-12, Islamabad, Pakistan \\ ${ }^{3}$ Department of Mathematics, University of Management and Technology, Sialkot, Pakistan \\ ${ }^{4}$ Department of Natural Sciences and Humanities, University of Engineering and Technology Lahore (RCET), Lahore, Pakistan \\ ${ }^{5}$ Faculty of Physical and Numerical Sciences, Abdul Wali Khan University, Mardan, Pakistan \\ Correspondence should be addressed to Muhammad Aamir; aamirkhan@awkum.edu.pk
}

Received 16 October 2020; Revised 18 December 2020; Accepted 24 December 2020; Published 18 January 2021

Academic Editor: Muhammad J. Habib

Copyright (c) 2021 Jialin Zheng et al. This is an open access article distributed under the Creative Commons Attribution License, which permits unrestricted use, distribution, and reproduction in any medium, provided the original work is properly cited.

\begin{abstract}
The study of graphs and networks accomplished by topological measures plays an applicable task to obtain their hidden topologies. This procedure has been greatly used in cheminformatics, bioinformatics, and biomedicine, where estimations based on graph invariants have been made available for effectively communicating with the different challenging tasks. Irregularity measures are mostly used for the characterization of the nonregular graphs. In several applications and problems in various areas of research like material engineering and chemistry, it is helpful to be well-informed about the irregularity of the underline structure. Furthermore, the irregularity indices of graphs are not only suitable for quantitative structure-activity relationship (QSAR) and quantitative structure-property relationship (QSPR) studies but also for a number of chemical and physical properties, including toxicity, enthalpy of vaporization, resistance, boiling and melting points, and entropy. In this article, we compute the irregularity measures including the variance of vertex degrees, the total irregularity index, the $\sigma$ irregularity index, and the Gini index of a new graph operation.
\end{abstract}

\section{Introduction}

Mathematical chemistry is showing assistance by providing the time-saving and competent tools for the characterizations of chemical compounds instead of any other data as input. Topological invariants catch some key topological and combinatorial details of the underline structures, and with the help of these, some properties of these structures can be deduced without the assistance of the quantum mechanics [1-3]. Topological invariants are numerical quantities that characterize various structural properties of an underline structure. In pharmacy, mathematical chemistry, and environmental sciences, these descriptors are used for the QSAR/QSPR studies, where biological or chemical activities and physical properties of an underline structure are associated with its molecular structure. Therefore, these descriptors are mostly quoted as molecular descriptors [4]. Furthermore, topological descriptors are becoming essential also in the theory of networks and complex systems [5]. More precisely, they are practised for quantifying numerous global and local properties in biological networks, social networks, neural networks, and communications networks. As a result, topological descriptors can be exercised in different scientific fields. However, molecular descriptors generally require to be computed of various families of molecules, and hence, algorithms and effective methods for the computation are required. Therefore, determining these numerical indices is one of the good-looking areas of research. Some well-known classes of numerical descriptors are distance-based, degree-based, and counting-related. Degree-based indices are the most frequently used invariants these days [4, 6-9]. Some correlations of the topological indices with the chemical reactivity, physical properties, or biological activity of compounds can be seen in [2, 3, 6-9]. In short, these invariants take into account the internal atomic arrangement of the compounds and yield several facts in the 
mathematical form about the multiple bonds, presence of heteroatoms, molecular size, branching, and shape. Among degree-based indices, irregularity measures may play an important role in chemistry, especially in the QSPR/QSAR studies $[1,10]$ as well as network theory [5, 11]. These measures have been chosen to analyse the topological aspects of the underline structures; for instance, see [5, 10-17].

In 1957, Collatz and Sinogowitz introduced the term "network irregularity" [18]. In fact, irregularity measures are used in complex networks, which speak on behalf of disparate systems. These systems have main topological features like self-similarity, scale-freeness, network motifs, and small worldness [5]. On the contrary, the molecular complexity has been discussed in the scientific literature from decades; due to its ever-increasing significance, one is interested to go through the process of estimating the degree of complexity. One way is to work out the irregularity measures of the underlined structures $[13,15,19,20]$. By using irregularity measures, Reti et al. examine the physicochemical properties, including an acentric factor of octane isomers entropy, the entropy of vaporization, boiling point, and standard enthalpy of vaporization [10]. Actually, they showed that, by using these irregularity measures, some properties of isomers can be estimated with excellent accuracy. Estrada examined the irregularity of various networks in distinct organisms and showed that these networks achieve higher irregularity as compared to some regular networks [11]. So, these facts provide motivation to study the irregularity of structures.

A graph will be a regular graph if all its vertices have the same degrees. If not, then it will be an irregular graph. Recently, the total irregularity index has been suggested, and concerning this index, some well-known graphs have been studied. Bell examined the irregularity of graphs by using different irregularity indices [13]. Gutman provided some basic results for some irregularity measures of graphs in [15].

Throughout this paper, all graphs will assume to be simple, finite, and connected. We represent the vertex set of a graph $Q$ with $V(Q)$ and edge set with $E(Q)$. The order and size of $Q$ are presented as $\mathfrak{p}$ and $\mathfrak{q}$, respectively. The degree of a vertex $q \in V(Q)$, denoted as $\mu_{Q}(q)$, is the number of linked vertices to $q$ in $Q$. A topological index is said to be an irregularity measure if this has a nonzero value for a nonregular graph, and it has a value equal to zero for a regular graph. The applications of regular graphs in chemical graph theory appeared after the discovery of fullerenes and nanotubes. The easiest manners of manifesting the irregularities are by using irregularity measures. Most of the irregularity measures used the idea of the imbalance of an edge $e=q q^{\prime} \in E(Q)$ specified as $\operatorname{imb}(e):=\left|\mu_{Q}(q)-\mu_{Q}\left(q^{\prime}\right)\right|$. In 1997, the term "The graph irregularity" was proposed by Albertson [19]. For a graph $Q$, it is symbolized by $\operatorname{irr}(Q)$ and detailed as follows:

$$
\operatorname{irr}(Q)=\sum_{e \in E(Q)} \operatorname{imb}(e) .
$$

This invariant is also familiar as the third Zagreb index. Albertson [19] presented some upper bounds of irregularity for trees and bipartite and triangle-free graphs. The connections between the irregularity of trees and unicyclic graphs with their respective matching numbers were explored in [21]. Hansen and Melot [22] described the graphs having the maximal irregularity index. Abdo and Dimitrov [23] computed the irregularity indices of some graph operations. The total irregularity index of a graph $Q$ was introduced in [24] in the following way:

$$
\operatorname{irr}_{t}(Q)=\frac{1}{2} \sum_{q, q^{\prime} \in V(Q)}\left|\mu_{Q}(q)-\mu_{Q}\left(q^{\prime}\right)\right|
$$

The characterization of graphs with respect to extremal irregularity and the relationships among the various irregularity measures are studied in [25]. By using the irregularity of graphs, Fath-Tabar [26] provided novel bounds of Zagreb indices. For the comprehensive study about these irregularity measures, we refer $[27,28]$. The $\sigma$ irregularity index of a graph $Q$ is introduced by Gutman et al. in [29] as follows:

$$
\sigma(Q)=\sum_{q q^{\prime} \in E(Q)}\left(\mu_{Q}(q)-\mu_{Q}\left(q^{\prime}\right)\right)^{2} .
$$

For different properties of this index, the interested reader may refer to $[30,31]$. If the size and order of $Q$ are $\mathfrak{q}$ and $\mathfrak{p}$, then the variance of $Q$ can be expressed as follows [13]:

$$
\operatorname{Var}(Q)=\frac{1}{\mathfrak{p}} \sum_{q \in V(Q)} \mu_{Q}^{2}(Q)-\frac{1}{\mathfrak{p}^{2}}\left(\sum_{q \in V(Q)} \mu_{Q}(Q)\right)^{2}
$$

Gini index of $Q$ is denoted by $\zeta(Q)$ and is defined as

$$
\zeta(Q)=\frac{1}{2 \mathfrak{p q}} \operatorname{irr}_{t}(Q) .
$$

In 1972, Gutman and Trinajestic [1] the first and second Zagreb indices as follows:

$$
\begin{aligned}
& M_{1}(Q)=\sum_{q \in V(Q)} \mu_{Q}^{2}(q), \\
& M_{2}(Q)=\sum_{q q^{\prime} \in E(Q)} \mu_{Q}(q) \mu_{Q}\left(q^{\prime}\right) .
\end{aligned}
$$

These indices have significant applications in chemistry and its related fields. The forgotten topological index of $Q$ was initiated by Furtula and Gutman in [32] in the following way:

$$
F(Q)=\sum_{q \in V(Q)} \mu_{Q}^{3}(q)
$$

Like Zagreb indices, the first $\mathrm{IRM}_{1}$ and the second $\mathrm{IRM}_{2}$ irregularity indices are as follows [33]:

$$
\begin{aligned}
& \operatorname{IRM}_{1}(Q)=\frac{1}{\mathfrak{p}} \sum_{q \in V(Q)} \eta_{Q}(q)-\frac{2 \mathfrak{q}}{\mathfrak{p}}, \\
& \operatorname{IRM}_{2}(Q)=\sum_{q \in V(Q)}\left|\mu_{Q}(q)-\eta_{Q}(q)\right|,
\end{aligned}
$$




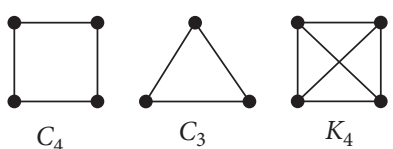

(a) (b)

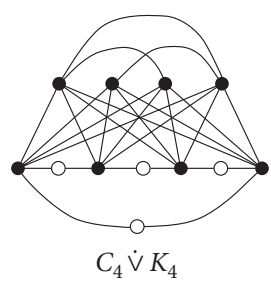

(d)
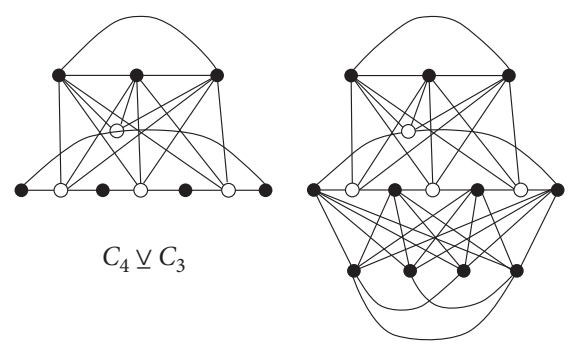

$C_{4}^{S} \triangleright\left(K_{4}^{V} \cup C_{3}^{E}\right)$

(f)

Figure 1: $C_{4} \dot{\vee} K_{4}, C_{4} \vee C_{3}$, and $C_{4}^{S} \triangleright\left(K_{4}^{V} \cup C_{3}^{E}\right)$.

where $\eta_{Q}(q)$ is the average of the degrees of the adjacent vertices of $q$.

A novel graph can be designed from the given graphs by using graph operations, and it is demonstrated that a number of chemical graphs can be constructed with the help of graph operations. The relations achieved for different topological descriptors of graph operations in the form of topological descriptors of their components; thus, it is valuable to find out the topological invariants of some molecular graphs and nanostructures. There are a number of studies about the topological indices of various graph operations; for instance, see [34-50]. In short space of time, novel graph operation, famous as the subdivision vertexedge join (SVE-join), is suggested in [51]. For a graph $Q$, let $S(Q)$ represents the subdivision graph of it, and the vertex set of $S(Q)$ can be divided into two parts: the first one is the $V(Q)$, and the second one, represented by $\mathscr{I}(Q)$, that contains the inserting vertices is related to the edges of $Q$. Let us consider two disjoint graphs $T$ and $Y$. The SVE-join of $Q$ with $T$ and $Y$, symbolized by $Q^{S} \triangleright\left(T^{V} \cup Y^{E}\right)$, is the graph consisting of $S(Q), T$ and $Y$, all vertex-disjoint, and joining the $i$-th vertex of $V(Q)$ to each vertex of $V(T)$ and $i$-th vertex of $\mathscr{I}(Q)$ to each vertex of $V(Y)$. For example, see Figure 1. It is easy to see that $Q^{S} \triangleright\left(T^{V} \cup Y^{E}\right)$ has order $\mathfrak{p}_{1}+\mathfrak{q}_{1}+\mathfrak{p}_{2}+\mathfrak{p}_{3}$ and its size is $2 \mathfrak{q}_{1}+\mathfrak{p}_{1} \mathfrak{p}_{2}+\mathfrak{q}_{1} \mathfrak{p}_{3}+\mathfrak{q}_{2}+\mathfrak{q}_{3}$, where $\mathfrak{p}_{1}$ and $\mathfrak{q}_{1}$ are the number of vertices and edges of $Q, \mathfrak{p}_{2}$ and $\mathfrak{q}_{2}$ are the order and size of $T$, and $\mathfrak{p}_{3}$ and $\mathfrak{q}_{3}$ are the order and size of $Y$.
Furthermore, we see that $Q^{S} \triangleright\left(T^{V} \cup Y^{E}\right)$ is $Q \dot{V} T$ if $Y$ is the null graph and is $Q \vee Y$ if $T$ is the null graph [52].

\section{Main Results}

In this section, first, by using the definition of SVE-join, we express several properties of the subdivision vertex-edge join of three graphs. After this, by applying these consequences, we provide our main results.

Lemma 1. For the graphs $Q, T$, and $Y$, we have the following:

$$
\begin{array}{r}
\text { (1) } \mu_{Q^{S} \triangleright\left(T^{V} \cup Y^{E}\right)}(q)= \begin{cases}\mu_{T}(q)+\mathfrak{p}_{1} & \text { if } q \in V(T) \\
\mu_{Y}(q)+\mathfrak{q}_{1} & \text { if } q \in V(Y) \\
\mu_{Q}(q)+\mathfrak{p}_{2} & \text { if } q \in V(Q) \\
\mathfrak{p}_{3}+2 & \text { if } q \in E(Q)\end{cases} \\
\text { (2) } \eta_{Q^{S_{\triangleright}\left(T^{V} \cup Y^{E}\right)}}(q)= \begin{cases}\mu_{T}(q)+\mathfrak{p}_{1} & \text { if } q \in V(T) \\
\mu_{Y}(q)+\mathfrak{q}_{1} & \text { if } q \in V(Y) \\
\mu_{Q}(q)+\mathfrak{p}_{2} & \text { if } q \in V(Q) \\
\mathfrak{p}_{3}+2 & \text { if } q \in E(Q)\end{cases}
\end{array}
$$

Here, $\omega_{T}(q)$ is the sum of degrees of its linked vertices in $T$ and $\omega_{Y}(q)$ is the sum of degrees of its linked vertices in $Y$.

Next, we give the expression of the variance of the SVEjoin of three graphs.

Theorem 1. For the graphs $Q, T$, and $Y$, we have the following:

$$
\begin{aligned}
\operatorname{Var}\left(Q^{S} \triangleright\left(T^{V} \cup Y^{E}\right)\right)= & \frac{1}{\mathfrak{p}_{1}+\mathfrak{p}_{2}+\mathfrak{p}_{3}+\mathfrak{q}_{1}} \\
& \cdot\left(M_{1}(Q)+M_{1}(T)+M_{1}(Y)+\mathfrak{p}_{1} \mathfrak{p}_{2}\left(\mathfrak{p}_{1}+\mathfrak{p}_{2}\right)+4\left(\mathfrak{p}_{2} \mathfrak{q}_{1}+\mathfrak{p}_{1} \mathfrak{q}_{2}\right)+\mathfrak{p}_{3} \mathfrak{q}_{1}\left(\mathfrak{q}_{1}+\mathfrak{p}_{3}\right)+4 \mathfrak{q}_{1}\left(1+\mathfrak{q}_{3}+\mathfrak{p}_{3}\right)\right. \\
& \left.-\frac{2\left(2 \mathfrak{q}_{1}+\mathfrak{q}_{2}+\mathfrak{q}_{3}+\mathfrak{p}_{1} \mathfrak{p}_{2}+\mathfrak{p}_{3} \mathfrak{q}_{1}\right)}{\mathfrak{p}_{1}+\mathfrak{p}_{2}+\mathfrak{p}_{3}+\mathfrak{q}_{1}}\right) .
\end{aligned}
$$

Proof. By using Lemma 1 in (4), we obtain 


$$
\begin{aligned}
& \operatorname{Var}\left(Q^{S} \triangleright\left(T^{V} \cup Y^{E}\right)\right)=\frac{1}{\mathfrak{p}_{1}+\mathfrak{p}_{2}+\mathfrak{p}_{3}+\mathfrak{q}_{1}} \sum_{q \in V\left(Q^{S} \triangleright\left(T^{V} \cup Y^{E}\right)\right)} \mu_{Q^{S} \triangleright\left(T^{V} \cup Y^{E}\right)}^{2}(Q) \\
& -\frac{1}{\left(\mathfrak{p}_{1}+\mathfrak{p}_{2}+\mathfrak{p}_{3}+\mathfrak{q}_{1}\right)^{2}}\left(\sum_{q \in V\left(Q^{\left.S \triangleright\left(T^{V} \cup Y^{E}\right)\right)}\right.} \mu_{Q^{S \triangleright\left(T^{V} \cup Y^{E}\right)}}(Q)\right)^{2} \\
& =\frac{1}{\mathfrak{p}_{1}+\mathfrak{p}_{2}+\mathfrak{p}_{2}+\mathfrak{q}_{1}}\left(\sum_{q \in V(Q)}\left(\mu_{Q}(Q)+\mathfrak{p}_{2}\right)^{2}+\sum_{q \in E(Q)}\left(\mathfrak{p}_{3}+2\right)^{2}+\sum_{q \in V(T)}\left(\mu_{T}(Q)+\mathfrak{p}_{1}\right)^{2}\right. \\
& \left.+\sum_{q \in V(Y)}\left(\mu_{Y}(Q)+\mathfrak{q}_{1}\right)^{2}\right)+\frac{1}{\left(\mathfrak{p}_{1}+\mathfrak{p}_{2}+\mathfrak{p}_{3}+\mathfrak{q}_{1}\right)^{2}}\left(\sum_{q \in V(Q)}\left(\mu_{Q}(Q)+\mathfrak{p}_{2}\right)+\sum_{q \in E(Q)}\left(\mathfrak{p}_{3}+2\right)\right. \\
& \left.+\sum_{q \in V(T)}\left(\mu_{T}(Q)+\mathfrak{p}_{1}\right)+\sum_{q \in V(Y)}\left(\mu_{Y}(Q)+\mathfrak{q}_{1}\right)\right)^{2} \\
& =\frac{1}{\mathfrak{p}_{1}+\mathfrak{p}_{2}+\mathfrak{p}_{3}+\mathfrak{q}_{1}}\left(M_{1}(Q)+\mathfrak{p}_{2}^{2} \mathfrak{p}_{1}+4 \mathfrak{p}_{2} \mathfrak{q}_{1}+\mathfrak{q}_{1}\left(\mathfrak{p}_{3}^{2}+4+4 \mathfrak{p}_{3}\right)+M_{1}(T)+\mathfrak{p}_{1}^{2} \mathfrak{p}_{2}+4 \mathfrak{p}_{1} \mathfrak{q}_{2}+M_{1}(Y)\right. \\
& \left.+\mathfrak{q}_{1}^{2} \mathfrak{p}_{3}+4 \mathfrak{q}_{1} \mathfrak{q}_{3}\right)+\frac{1}{\left(\mathfrak{p}_{1}+\mathfrak{p}_{2}+\mathfrak{p}_{3}+\mathfrak{q}_{1}\right)^{2}}\left(2 \mathfrak{q}_{1}+\mathfrak{p}_{1} \mathfrak{p}_{2}+\mathfrak{q}_{1}\left(\mathfrak{p}_{3}+2\right)+2 \mathfrak{q}_{2}+\mathfrak{p}_{1} \mathfrak{p}_{2}+2 \mathfrak{q}_{3}+\mathfrak{q}_{1} \mathfrak{p}_{3}\right) \\
& =\frac{1}{\mathfrak{p}_{1}+\mathfrak{p}_{2}+\mathfrak{p}_{3}+\mathfrak{q}_{1}}\left(M_{1}(Q)+M_{1}(T)+M_{1}(Y)+\mathfrak{p}_{1} \mathfrak{p}_{2}\left(\mathfrak{p}_{1}+\mathfrak{p}_{2}\right)+4\left(\mathfrak{p}_{2} \mathfrak{q}_{1}+\mathfrak{p}_{1} \mathfrak{q}_{2}\right)+\mathfrak{p}_{3} \mathfrak{q}_{1}\left(\mathfrak{q}_{1}+\mathfrak{p}_{3}\right)\right. \\
& \left.+4 \mathfrak{q}_{1}\left(1+\mathfrak{q}_{3}+\mathfrak{p}_{3}\right)-\frac{2\left(2 \mathfrak{q}_{1}+\mathfrak{q}_{2}+\mathfrak{q}_{3}+\mathfrak{p}_{1} \mathfrak{p}_{2}+\mathfrak{p}_{3} \mathfrak{q}_{1}\right)}{\mathfrak{p}_{1}+\mathfrak{p}_{2}+\mathfrak{p}_{3}+\mathfrak{q}_{1}}\right)
\end{aligned}
$$

This completes the proof.

Theorem 2. For the graphs $Q, T$, and $Y$, we have the following:

We proceed further to construct an upper bound of the total irregularity index of the SVE-join of three graphs in terms of their orders and sizes.

$$
\begin{aligned}
\operatorname{irr}_{t}\left(Q^{S} \triangleright\left(T^{V} \cup Y^{E}\right)\right) \leq & \operatorname{irr}_{t}(Q)+\operatorname{irr}_{t}(T)+\operatorname{irr}_{t}(Y)+\frac{\mathfrak{p}_{1} \mathfrak{p}_{2}}{2}\left(\mathfrak{p}_{2}-\mathfrak{p}_{1}\right)+\mathfrak{p}_{1} \mathfrak{p}_{3}\left(\mathfrak{p}_{2}+\mathfrak{q}_{1}\right)+\mathfrak{p}_{2} \mathfrak{q}_{1}\left(\mathfrak{p}_{1}-\mathfrak{p}_{3}\right) \\
& +\frac{\mathfrak{p}_{3} \mathfrak{q}_{1}}{2}\left(\mathfrak{p}_{3}-\mathfrak{q}_{1}\right)+\mathfrak{q}_{1}\left(\mathfrak{p}_{2}+\mathfrak{q}_{1}-\mathfrak{p}_{1}+2 \mathfrak{p}_{3}\right)+\mathfrak{q}_{2}\left(\mathfrak{p}_{1}+\mathfrak{q}_{1}+\mathfrak{p}_{3}\right)+\mathfrak{q}_{3}\left(\mathfrak{q}_{1}+\mathfrak{p}_{2}+\mathfrak{p}_{1}\right)
\end{aligned}
$$


Proof. By using Lemma 1 in (2), its follows that

$$
\begin{aligned}
& \operatorname{irr}_{t}\left(Q^{S} \triangleright\left(T^{V} \cup Y^{E}\right)\right)=\frac{1}{2} \sum_{q, q^{\prime} \in V\left(Q^{S} \triangleright\left(T^{V} \cup Y^{E}\right)\right)}\left|\mu_{Q^{S} \triangleright\left(T^{V} \cup Y^{E}\right)}(q)-\mu_{Q^{S}\left(T^{V} \cup Y^{E}\right)}\left(q^{\prime}\right)\right| \\
& =\frac{1}{2} \sum_{q, q^{\prime} \in V(T)}\left|\mu_{T}(q)+\mathfrak{p}_{1}-\mu_{T}\left(q t^{\prime}\right)-\mathfrak{p}_{1}\right|+\frac{1}{2} \sum_{q, q^{\prime} \in V(Y)}\left|\mu_{Y}(q)+\mathfrak{q}_{1}-\mu_{Y}\left(q^{\prime}\right)-\mathfrak{q}_{1}\right| \\
& +\frac{1}{2} \sum_{q, q^{\prime} \in V(Q)}\left|\mu_{Q}(q)+\mathfrak{p}_{2}-\mu_{Q}\left(q^{\prime}\right)-\mathfrak{p}_{2}\right|+\frac{1}{2} \sum_{q, q^{\prime} \in E(Q)}\left|\mathfrak{p}_{3}+2-\mathfrak{p}_{3}-2\right| \\
& +\frac{1}{2} \sum_{q \in V(Q)} \sum_{q^{\prime} \in V(T)}\left|\mu_{Q}(q)+\mathfrak{p}_{2}-\mu_{T}\left(q^{\prime}\right)-\mathfrak{p}_{1}\right| \\
& +\frac{1}{2} \sum_{q \in V(Q)} \sum_{q^{\prime} \in V(Y)}\left|\mu_{Q}(q)+\mathfrak{p}_{2}-\mu_{Y}\left(q^{\prime}\right)-\mathfrak{q}_{1}\right|+\frac{1}{2} \sum_{q \in V(Q)} \sum_{q^{\prime} \in E(Q)}\left|\mu_{Q}(q)+\mathfrak{p}_{2}-\mathfrak{p}_{3}-2\right| \\
& +\frac{1}{2} \sum_{q \in V(T)} \sum_{q^{\prime} \in E(Q)}\left|\mu_{T}(q)+\mathfrak{q}_{1}-\mathfrak{p}_{3}-2\right|+\frac{1}{2} \sum_{q \in V(T)} \sum_{q^{\prime} \in V(Y)}\left|\mu_{T}(q)+\mathfrak{p}_{1}-\mu_{Y}\left(q^{\prime}\right)-\mathfrak{q}_{1}\right| \\
& +\frac{1}{2} \sum_{q \in E(Q)} \sum_{q^{\prime} \in V(Y)}\left|\mathfrak{p}_{3}+2-\mu_{Y}\left(q^{\prime}\right)-\mathfrak{q}_{1}\right| \leq \operatorname{irr}_{t}(Q)+\operatorname{irr}_{t}(T)+\operatorname{irr}_{t}(Y)+0+\frac{1}{2} \sum_{q \in V(Q)} \sum_{q^{\prime} \in V(T)} \mu_{Q}(q) \\
& +\frac{1}{2} \sum_{q \in V(Q)} \sum_{q^{\prime} \in V(T)} \mu_{T}\left(q^{\prime}\right)+\frac{1}{2} \sum_{q \in V(Q)} \sum_{q^{\prime} \in V(T)}\left(\mathfrak{p}_{2}-\mathfrak{p}_{1}\right)+\frac{1}{2} \sum_{q \in V(Q)} \sum_{q^{\prime} \in V(Y)} \mu_{Q}(q) \\
& +\frac{1}{2} \sum_{q \in V(Q)} \sum_{q^{\prime} \in V(Y)} \mu_{Y}\left(q^{\prime}\right)+\frac{1}{2} \sum_{q \in V(Q)} \sum_{q^{\prime} \in V(Y)}\left(\mathfrak{p}_{2}-\mathfrak{q}_{1}\right)+\frac{1}{2} \sum_{q \in V(Q)} \sum_{q^{\prime} \in E(Q)} \mu_{Q}(q) \\
& +\frac{1}{2} \sum_{q \in V(Q)} \sum_{q^{\prime} \in E(Q)}\left(\mathfrak{p}_{2}-\mathfrak{p}_{3}-2\right)+\frac{1}{2} \sum_{q \in V(T)} \sum_{q^{\prime} \in E(Q)} \mu_{T}(q)+\frac{1}{2} \sum_{q \in V(T)} \sum_{q^{\prime} \in E(Q)}\left(\mathfrak{q}_{1}-\mathfrak{p}_{3}-2\right) \\
& +\frac{1}{2} \sum_{q \in V(T)} \sum_{q^{\prime} \in V(Y)} \mu_{T}(q)+\frac{1}{2} \sum_{q \in V(T)} \sum_{q^{\prime} \in V(Y)} \mu_{Y}\left(q^{\prime}\right)+\frac{1}{2} \sum_{q \in V(T)} \sum_{q^{\prime} \in V(Y)}\left(\mathfrak{p}_{1}-\mathfrak{q}_{1}\right) \\
& +\frac{1}{2} \sum_{q \in E(Q)} \sum_{q^{\prime} \in V(Y)} \mu_{Y}\left(q^{\prime}\right)+\frac{1}{2} \sum_{q \in E(Q)} \sum_{q^{\prime} \in V(Y)}\left(\mathfrak{p}_{3}+2-\mathfrak{q}_{1}\right) \\
& =\operatorname{irr}_{t}(Q)+\operatorname{irr}_{t}(T)+\operatorname{irr}_{t}(Y)+\mathfrak{p}_{2} \mathfrak{q}_{1}+\mathfrak{p}_{1} \mathfrak{q}_{2}+\frac{\mathfrak{p}_{1} \mathfrak{p}_{2}}{2}\left(\mathfrak{p}_{2}-\mathfrak{p}_{1}\right)+\mathfrak{q}_{1} \mathfrak{p}_{3}+\mathfrak{p}_{1} \mathfrak{q}_{3}+\frac{\mathfrak{p}_{1} \mathfrak{p}_{3}}{2}\left(\mathfrak{p}_{2}-\mathfrak{q}_{1}\right) \\
& +\mathfrak{q}_{1}^{2}+\frac{\mathfrak{p}_{1} \mathfrak{q}_{1}}{2}\left(\mathfrak{p}_{2}-\mathfrak{p}_{3}-2\right)+\mathfrak{q}_{1} \mathfrak{p}_{2}+\frac{\mathfrak{q}_{1} \mathfrak{p}_{2}}{2}\left(\mathfrak{p}_{1}-\mathfrak{p}_{3}-2\right)+\mathfrak{q}_{1} \mathfrak{q}_{2}+\frac{\mathfrak{p}_{2} \mathfrak{p}_{3}}{2}\left(\mathfrak{p}_{1}-\mathfrak{q}_{1}\right)+\mathfrak{q}_{2} \mathfrak{p}_{3}+\mathfrak{q}_{3} \mathfrak{p}_{2} \\
& +\frac{\mathfrak{q}_{1} \mathfrak{p}_{3}}{2}\left(\mathfrak{p}_{3}+2-\mathfrak{q}_{1}\right)+\mathfrak{q}_{1} \mathfrak{q}_{3}
\end{aligned}
$$


Result follows from simplifications.

Corollary 1. For the graphs $Q, T$, and $Y$, we have

In the upcoming corollary, we give an upper bound of Gini index of $Q^{S} \triangleright\left(T^{V} \cup Y^{E}\right)$. The proof follows from Theorem 2 and equation (5) and hence omitted.

$$
\begin{aligned}
\zeta\left(Q^{S} \triangleright\left(T^{V} \cup Y^{E}\right)\right) \leq & \frac{1}{2\left(\mathfrak{p}_{1}+\mathfrak{p}_{2}+\mathfrak{p}_{3}+\mathfrak{q}_{1}\right)\left(2 \mathfrak{q}_{1}+\mathfrak{q}_{2}+\mathfrak{q}_{3}+\mathfrak{p}_{1} \mathfrak{p}_{2}+\mathfrak{p}_{3} \mathfrak{q}_{1}\right)}\left(\operatorname{irr}_{t}(Q)+\operatorname{irr}_{t}(T)+\operatorname{irr}_{t}(Y)\right. \\
& +\frac{\mathfrak{p}_{1} \mathfrak{p}_{2}}{2}\left(\mathfrak{p}_{2}-\mathfrak{p}_{1}\right)+\mathfrak{p}_{1} \mathfrak{p}_{3}\left(\mathfrak{p}_{2}+\mathfrak{q}_{1}\right)+\mathfrak{p}_{2} \mathfrak{q}_{1}\left(\mathfrak{p}_{1}-\mathfrak{p}_{3}\right)+\frac{\mathfrak{p}_{3} \mathfrak{q}_{1}}{2}\left(\mathfrak{p}_{3}-\mathfrak{q}_{1}\right)+\mathfrak{q}_{1}\left(\mathfrak{p}_{2}+\mathfrak{q}_{1}\right. \\
& \left.\left.-\mathfrak{p}_{1}+2 \mathfrak{p}_{3}\right)+\mathfrak{q}_{2}\left(\mathfrak{p}_{1}+\mathfrak{q}_{1}+\mathfrak{p}_{3}\right)+\mathfrak{q}_{3}\left(\mathfrak{q}_{1}+\mathfrak{p}_{2}+\mathfrak{p}_{1}\right)\right) .
\end{aligned}
$$

In the next result, we find the formula of $\sigma$ index for the subdivision vertex-edge join of three graphs in terms of their orders and sizes.

Theorem 3. For the graphs $Q, T$, and $Y$, we have

$$
\begin{aligned}
\sigma\left(Q^{S} \triangleright\left(T^{V} \cup Y^{E}\right)\right)= & \sigma(T)+\sigma(Y)+F(Q)+\left(3 \mathfrak{p}_{2}-2 \mathfrak{p}_{3}-4\right) M_{1}(Q)+\mathfrak{p}_{1} M_{1}(T)+\mathfrak{q}_{1} M_{1}(Y) \\
& +\mathfrak{p}_{1} \mathfrak{p}_{2}\left(\mathfrak{p}_{2}-\mathfrak{p}_{1}\right)^{2}-8 \mathfrak{q}_{1} \mathfrak{q}_{2}+4\left(\mathfrak{p}_{2}-\mathfrak{p}_{1}\right)\left(\mathfrak{p}_{2} \mathfrak{q}_{1}-\mathfrak{p}_{1} \mathfrak{q}_{2}\right)-4 \mathfrak{q}_{1} \mathfrak{q}_{3}\left(\mathfrak{p}_{3}-\mathfrak{q}_{1}+2\right) \\
& +2 \mathfrak{q}_{1} \mathfrak{p}_{3}\left(3 \mathfrak{p}_{3}-2 \mathfrak{q}_{1}+6\right)+\mathfrak{q}_{1} \mathfrak{p}_{3}\left(\mathfrak{p}_{3}-\mathfrak{q}_{1}\right)^{2}+2 \mathfrak{q}_{1}\left(\mathfrak{p}_{2}-2\right)^{2}-4 \mathfrak{p}_{2} \mathfrak{p}_{3} \mathfrak{q}_{1} .
\end{aligned}
$$

Proof. By using Lemma 1 in (3), it follows that

$$
\begin{aligned}
\sigma\left(Q^{S} \triangleright\left(T^{V} \cup Y^{E}\right)=\right. & \sum_{q q^{\prime} \in E\left(Q^{S} \triangleright\left(T^{V} \cup Y^{E}\right)\right)}\left(\mu_{Q^{S} \triangleright\left(T^{V} \cup Y^{E}\right)}(Q)-\mu_{Q^{S} \triangleright\left(T^{V} \cup Y^{E}\right)}\left(q^{\prime}\right)^{2}\right) \\
= & \sum_{q q^{\prime} \in E(T)}\left(\mu_{T}(Q)+\mathfrak{p}_{1}-\mu_{T}\left(q^{\prime}\right)-\mathfrak{p}_{1}\right)^{2}+\sum_{q q^{\prime} \in E(Y)}\left(\mu_{Y}(Q)+\mathfrak{q}_{1}-\mu_{Y}\left(q^{\prime}\right)-\mathfrak{q}_{1}\right)^{2} \\
& +\sum_{q \in V(Q)} \sum_{q^{\prime} \in V(T)}\left(\mu_{Q}(Q)+\mathfrak{p}_{2}-\mu_{T}\left(q^{\prime}\right)-\mathfrak{p}_{1}\right)^{2} \\
& +\sum_{q \in E(Q)} \sum_{q^{\prime} \in V(Y)}\left(\mathfrak{p}_{3}+2-\mu_{Y}\left(q^{\prime}\right)-\mathfrak{q}_{1}\right)^{2}+\sum_{q \in V(Q)} \sum_{q^{\prime} \in E(Q)}\left(\mu_{Q}(Q)+\mathfrak{p}_{2}-\mathfrak{p}_{3}-2\right)^{2} \\
= & \sum_{q q^{\prime} \in E(T)}\left(\mu_{T}(Q)-\mu_{T}\left(q^{\prime}\right)^{2}+\sum_{q q^{\prime} \in E(Y)}\left(\mu_{Y}(Q)-\mu_{Y}\left(q^{\prime}\right)^{2}\right)\right. \\
& +\sum_{q \in V(Q)} \sum_{q^{\prime} \in V(T)}\left(\mu_{Q}^{2}(Q)+\mu_{T}^{2}\left(q^{\prime}\right)+\left(\mathfrak{p}_{2}-\mathfrak{p}_{1}\right)^{2}-2 \mu_{Q}(Q) \mu_{T}\left(q^{\prime}\right)\right. \\
& \left.+2\left(\mathfrak{p}_{2}-\mathfrak{p}_{1}\right) \mu_{Q}(Q)-2\left(\mathfrak{p}_{2}-\mathfrak{p}_{1}\right) \mu_{T}\left(q^{\prime}\right)\right)+\sum_{q \in E(Q)} \sum_{q^{\prime} \in V(Y)}\left(\left(\mathfrak{p}_{3}+2-\mathfrak{q}_{1}\right)^{2}\right.
\end{aligned}
$$




$$
\begin{aligned}
& \left.+\mu_{Y}^{2}\left(q^{\prime}\right)-2\left(\mathfrak{p}_{3}+2-\mathfrak{q}_{1}\right) \mu_{Y}\left(q^{\prime}\right)\right)+\sum_{q \in V(Q)} \mu_{Q}(Q)\left(\mu_{Q}^{2}(Q)\right) \\
& \left.+\left(\mathfrak{p}_{2}-\mathfrak{p}_{3}-2\right)^{2}+2\left(\mathfrak{p}_{2}-\mathfrak{p}_{3}-2\right) \mu_{Q}(Q)\right) \\
= & \sigma(T)+\sigma(Y)+\sum_{q \in V(Q)} \sum_{q^{\prime} \in V(T)} \mu_{Q}^{2}(Q)+\sum_{q \in V(Q)} \sum_{q^{\prime} \in V(T)} \mu_{Q}^{2}(Q) \\
& +\mathfrak{p}_{1} \mathfrak{p}_{2}\left(\mathfrak{p}_{2}-\mathfrak{p}_{1}\right)^{2}-2 \sum_{q \in V(Q)} \mu_{Q}(Q) \sum_{q^{\prime} \in V(T)} \mu_{T}\left(q^{\prime}\right)+2\left(\mathfrak{p}_{2}-\mathfrak{p}_{1}\right) \\
& \cdot \sum_{q \in V(Q)} \sum_{q^{\prime} \in V(T)} \mu_{Q}(Q)-2\left(\mathfrak{p}_{2}-\mathfrak{p}_{1}\right) \sum_{q \in V(Q)} \sum_{q^{\prime} \in V(T)} \mu_{T}\left(q^{\prime}\right) \\
& +\mathfrak{q}_{1} \mathfrak{p}_{3}\left(\mathfrak{p}_{3}+2-\mathfrak{q}_{1}\right)^{2}+\sum_{q \in E(Q)} \sum_{q^{\prime} \in V(Y)} \mu_{Y}^{2}\left(q^{\prime}\right)-2\left(\mathfrak{p}_{3}+2-\mathfrak{q}_{1}\right) \\
& \cdot \sum_{q q \in E(Q)} \sum_{q^{\prime} \in V(Y)} \mu_{Y}\left(q^{\prime}\right)+\sum_{q \in V(Q)} \mu_{Q}^{3}(Q)+\left(\mathfrak{p}_{2}-\mathfrak{p}_{3}-2\right)^{2} \\
& \cdot \sum_{q \in V(Q)} \mu_{Q}(Q)+2\left(\mathfrak{p}_{2}-\mathfrak{p}_{3}-2\right) \sum_{q \in V(Q)} \mu_{Q}^{2}(Q) \\
= & \sigma(T)+\sigma(Y)+\mathfrak{p}_{2} M_{1}(Q)+\mathfrak{p}_{1} M_{1}(T)+\mathfrak{p}_{1} \mathfrak{p}_{2}\left(\mathfrak{p}_{2}-\mathfrak{p}_{1}\right)^{2}-2\left(2 \mathfrak{q}_{1}\right)\left(2 \mathfrak{q}_{2}\right) \\
& +2\left(\mathfrak{p}_{2}-\mathfrak{p}_{1}\right)\left(2 \mathfrak{q}_{1}\right)\left(\mathfrak{p}_{2}\right)-2\left(\mathfrak{p}_{2}-\mathfrak{p}_{1}\right)\left(2 \mathfrak{q}_{2}\right)\left(\mathfrak{p}_{1}\right)+\mathfrak{q}_{1} \mathfrak{p}_{3}\left(\mathfrak{p}_{3}+2-\mathfrak{q}_{1}\right)^{2}+\mathfrak{q}_{1} M_{1}(Y) \\
& 2\left(\mathfrak{p}_{3}+2-\mathfrak{q}_{1}\right) \mathfrak{q}_{1}\left(2 \mathfrak{q}_{3}\right)+F(Q)+2 \mathfrak{q}_{1}\left(\mathfrak{p}_{2}-\mathfrak{p}_{3}-2\right)^{2}+2\left(\mathfrak{p}_{2}-\mathfrak{p}_{3}-2\right) M_{1}(Q) .
\end{aligned}
$$

Result follows after some simplifications.

Next, we determine the expression for the $\mathrm{IRM}_{1}$ index of $Q^{S} \triangleright\left(T^{V} \cup Y^{E}\right)$.
Theorem 4. Let $Q, T$, and $Y$ be the three graphs. Then,

$$
\begin{aligned}
\operatorname{IRM}_{1}\left(Q^{S} \triangleright\left(T^{V} \cup Y^{E}\right)\right) \leq & \Gamma(T)+\Gamma(Y)+\left(2 \mathfrak{q}_{2}+\mathfrak{p}_{1} \mathfrak{p}_{2}\right) I D(Q)+\left(2 \mathfrak{q}_{1}+\mathfrak{p}_{1} \mathfrak{p}_{2}\right) I D(T)+\mathfrak{q}_{1}\left(\mathfrak{p}_{3}+2\right) \\
& \cdot I D(Y)+\frac{1}{\mathfrak{p}_{3}+2} M_{1}(Q)-\mathfrak{p}_{1} \mathfrak{p}_{2}-\frac{\mathfrak{q}_{1} \mathfrak{p}_{3}\left(\mathfrak{p}_{3}+2-\mathfrak{q}_{1}\right)}{\mathfrak{p}_{3}+2}+\frac{2 \mathfrak{q}_{1}\left(\mathfrak{p}_{2}+\mathfrak{q}_{3}\right)}{\mathfrak{p}_{3}+2} \\
& +\mathfrak{p}_{1}\left(\mathfrak{p}_{3}+2\right)-2\left(2 \mathfrak{q}_{1}+\mathfrak{q}_{2}+\mathfrak{q}_{3}\right),
\end{aligned}
$$


where $\quad \Gamma(T)=\sum_{q \in V(T)}\left(\omega_{T}(q) / \mu_{T}(q)\right) \quad$ and $\quad \Gamma(Y)=\quad$ Proof. By using Lemma 1 in (8), we obtain $\sum_{q \in V(Y)}\left(\omega_{Y}(q) / \mu_{Y}(q)\right)$.

$$
\begin{aligned}
\operatorname{IRM}_{1}\left(Q^{S} \triangleright\left(T^{V} \cup Y^{E}\right)\right)= & \frac{1}{\mathfrak{p}_{1}+\mathfrak{p}_{2}+\mathfrak{p}_{3}+\mathfrak{q}_{1}} \sum_{q \in V\left(Q^{S} \triangleright\left(T^{V} \cup Y^{E}\right)\right)} \eta_{Q^{S} \triangleright\left(T^{V} \cup Y^{E}\right)}(Q) \\
& -\frac{2\left(2 \mathfrak{q}_{1}+\mathfrak{p}_{1} \mathfrak{p}_{2}+\mathfrak{q}_{1} \mathfrak{p}_{3}+\mathfrak{q}_{2}+\mathfrak{q}_{3}\right)}{\mathfrak{p}_{1}+\mathfrak{p}_{2}+\mathfrak{p}_{3}+\mathfrak{q}_{1}} \\
= & \frac{1}{\mathfrak{p}_{1}+\mathfrak{p}_{2}+\mathfrak{p}_{3}+\mathfrak{q}_{1}}\left(\sum_{q \in V(T)} \frac{\omega_{T}(Q)+\mathfrak{p}_{1} \mu_{T}(Q)+2 \mathfrak{q}_{1}+\mathfrak{p}_{1} \mathfrak{p}_{2}}{\mu_{T}(Q)+\mathfrak{p}_{1}}\right. \\
& +\sum_{q \in V(Y)} \frac{\omega_{Y}(Q)+\mathfrak{q}_{1} \mu_{Y}(Q)+\mathfrak{q}_{1}\left(\mathfrak{p}_{3}+2\right)}{\mu_{Y}(Q)+\mathfrak{q}_{1}} \\
& +\sum_{q \in V(Q)} \frac{\mu_{Q}(Q)\left(\mathfrak{p}_{3}+2\right)+2 \mathfrak{q}_{2}+\mathfrak{p}_{1} \mathfrak{p}_{2}}{\mu_{Q}(Q)+\mathfrak{p}_{2}} \\
& \left.+\sum_{q=q q^{\prime} \in E(Q)} \frac{\mu_{Q}(Q)+\mu_{Q}\left(q^{\prime}\right)+2 \mathfrak{p}_{2}+2 \mathfrak{q}_{3}+\mathfrak{q}_{1} \mathfrak{p}_{3}}{\mathfrak{p}_{3}+2}\right) \\
& -\frac{2\left(2 \mathfrak{q}_{1}+\mathfrak{p}_{1} \mathfrak{p}_{2}+\mathfrak{q}_{1} \mathfrak{p}_{3}+\mathfrak{q}_{2}+\mathfrak{q}_{3}\right)}{\mathfrak{p}_{1}+\mathfrak{p}_{2}+\mathfrak{p}_{3}+\mathfrak{q}_{1}} .
\end{aligned}
$$

Since $\quad\left(1 / \mu_{T}(q)+\mathfrak{p}_{1}\right) \leq\left(1 / \mu_{T}(q)\right), \quad\left(1 / \mu_{Y}\left(q+\mathfrak{q}_{1}\right)\right) \leq$ $\left(1 / \mu_{Y}(q)\right)$ and $\left(1 / \mu_{Q}(q)+\mathfrak{p}_{2}\right) \leq\left(1 / \mu_{Q}(q)\right)$. Therefore,

$$
\begin{aligned}
& \operatorname{IRM}_{1}\left(Q^{S} \triangleright\left(T^{V} \cup Y^{E}\right)\right) \leq \frac{1}{\mathfrak{p}_{1}+\mathfrak{p}_{2}+\mathfrak{p}_{3}+\mathfrak{q}_{1}}\left(\sum_{q \in V(T)}\left(\frac{\omega_{T}(Q)}{\mu_{T}(Q)}+\mathfrak{p}_{1}+\left(2 \mathfrak{q}_{1}+\mathfrak{p}_{1} \mathfrak{p}_{2}\right) \frac{1}{\mu_{T}(Q)}\right)\right. \\
& +\sum_{q \in V(Y)}\left(\frac{\omega_{Y}(Q)}{\mu_{Y}(Q)}+\mathfrak{q}_{1}+\mathfrak{q}_{1}\left(\mathfrak{p}_{3}+2\right) \frac{1}{\mu_{Y}(Q)}\right)+\sum_{q \in V(Q)}\left(\left(\mathfrak{p}_{3}+2\right)\right. \\
& \left.+\left(2 \mathfrak{q}_{2}+\mathfrak{p}_{1} \mathfrak{p}_{2}\right) \frac{1}{\mu_{Q}(Q)}\right)+\frac{1}{\mathfrak{p}_{3}+2} \sum_{q=q q^{\prime} \in E(Q)}\left(\mu_{Q}(Q)+\mu_{Q}\left(q^{\prime}\right)\right. \\
& \left.\left.+2 \mathfrak{p}_{2}+2 \mathfrak{q}_{3}+\mathfrak{q}_{1} \mathfrak{p}_{3}\right)\right)-\frac{2\left(2 \mathfrak{q}_{1}+\mathfrak{p}_{1} \mathfrak{p}_{2}+\mathfrak{q}_{1} \mathfrak{p}_{3}+\mathfrak{q}_{2}+\mathfrak{q}_{3}\right)}{\mathfrak{p}_{1}+\mathfrak{p}_{2}+\mathfrak{p}_{3}+\mathfrak{q}_{1}} \\
& =\frac{1}{\mathfrak{p}_{1}+\mathfrak{p}_{2}+\mathfrak{p}_{3}+\mathfrak{q}_{1}}\left(\sum_{q \in V(T)} \frac{\omega_{T}(Q)}{\mu_{T}(Q)}+\sum_{q \in V(T)} \mathfrak{p}_{1}+\left(2 \mathfrak{q}_{1}+\mathfrak{p}_{1} \mathfrak{p}_{2}\right) \sum_{q \in V(T)} \frac{1}{\mu_{T}(Q)}\right. \\
& +\sum_{q \in V(Y)} \frac{\omega_{Y}(Q)}{\mu_{Y}(Q)}+\sum_{q \in V(Y)} \mathfrak{q}_{1}+\mathfrak{q}_{1}\left(\mathfrak{p}_{3}+2\right) \sum_{q \in V(Y)} \frac{1}{\mu_{Y}(Q)}+\sum_{q \in V(Q)}\left(\mathfrak{p}_{3}+2\right) \\
& +\left(2 \mathfrak{q}_{2}+\mathfrak{p}_{1} \mathfrak{p}_{2}\right) \sum_{q \in V(Q)} \frac{1}{\mu_{Q}(Q)}+\frac{1}{\mathfrak{p}_{3}+2} \sum_{q=q q^{\prime} \in E(Q)}\left(\mu_{Q}(Q)+\mu_{Q}\left(q^{\prime}\right)\right)
\end{aligned}
$$




$$
\begin{aligned}
& \left.+\frac{1}{\mathfrak{p}_{3}+2} \sum_{q=q q^{\prime} \in E(Q)}\left(2 \mathfrak{p}_{2}+2 \mathfrak{q}_{3}+\mathfrak{q}_{1} \mathfrak{p}_{3}\right)\right)-\frac{2\left(2 \mathfrak{q}_{1}+\mathfrak{p}_{1} \mathfrak{p}_{2}+\mathfrak{q}_{1} \mathfrak{p}_{3}+\mathfrak{q}_{2}+\mathfrak{q}_{3}\right)}{\mathfrak{p}_{1}+\mathfrak{p}_{2}+\mathfrak{p}_{3}+\mathfrak{q}_{1}} \\
= & \frac{1}{\mathfrak{p}_{1}+\mathfrak{p}_{2}+\mathfrak{p}_{3}+\mathfrak{q}_{1}}\left(\Gamma(T)+\mathfrak{p}_{1} \mathfrak{p}_{2}+\left(2 \mathfrak{q}_{1}+\mathfrak{p}_{1} \mathfrak{p}_{2}\right) I D(T)+\Gamma(Y)+\mathfrak{p}_{3} \mathfrak{q}_{1}\right. \\
& +\mathfrak{q}_{1}\left(\mathfrak{p}_{3}+2\right) I D(Y)+\mathfrak{p}_{1}\left(\mathfrak{p}_{3}+2\right)+\left(2 \mathfrak{q}_{2}+\mathfrak{p}_{1} \mathfrak{p}_{2}\right) I D(Q)+\frac{1}{\mathfrak{p}_{3}+2} M_{1}(Q) \\
& \left.+\frac{\mathfrak{q}_{1}\left(2 \mathfrak{p}_{2}+2 \mathfrak{q}_{3}+\mathfrak{q}_{1} \mathfrak{p}_{3}\right)}{\mathfrak{p}_{3}+2}-4 \mathfrak{q}_{1}-2 \mathfrak{p}_{1} \mathfrak{p}_{2}-2 \mathfrak{q}_{1} \mathfrak{p}_{3}-2 \mathfrak{q}_{2}-2 \mathfrak{q}_{3}\right) \\
= & \frac{1}{\mathfrak{p}_{1}+\mathfrak{p}_{2}+\mathfrak{p}_{3}+\mathfrak{q}_{1}}\left(\Gamma(T)+\Gamma(Y)+\left(2 \mathfrak{q}_{2}+\mathfrak{p}_{1} \mathfrak{p}_{2}\right) I D(Q)+\left(2 \mathfrak{q}_{1}+\mathfrak{p}_{1} \mathfrak{p}_{2}\right) I D(T)\right. \\
& +\mathfrak{q}_{1}\left(\mathfrak{p}_{3}+2\right) I D(Y)+\frac{1}{\mathfrak{p}_{3}+2} M_{1}(Q)-\mathfrak{p}_{1} \mathfrak{p}_{2}-\mathfrak{p}_{3} \mathfrak{q}_{1}\left(1-\frac{\mathfrak{q}_{1}}{\mathfrak{p}_{3}+2}\right)+\frac{2 \mathfrak{q}_{1}\left(\mathfrak{p}_{2}+\mathfrak{q}_{3}\right)}{\mathfrak{p}_{3}+2} \\
& \left.+\mathfrak{p}_{1}\left(\mathfrak{p}_{3}+2\right)-2\left(2 \mathfrak{q}_{1}-\mathfrak{q}_{2}-\mathfrak{q}_{3}\right)\right) .
\end{aligned}
$$

Result follows after some simplifications.

Theorem 5. For the graphs $Q, T$, and $Y$, we have

Finally, we give an upper bound for the $\mathrm{IRM}_{2}$ index of the subdivision vertex-edge join of $Q, T$, and $Y$.

$$
\begin{aligned}
\operatorname{IRM}_{2}\left(Q^{S} \triangleright\left(T^{V} \cup Y^{E}\right)\right) \leq & \Gamma(T)+\Gamma(Y)+\left(2 \mathfrak{q}_{1}+\mathfrak{p}_{1} \mathfrak{p}_{2}\right) I D(T)+\mathfrak{q}_{1}\left(\mathfrak{p}_{3}+2\right) I D(Y) \\
& +\left(2 \mathfrak{q}_{2}+\mathfrak{p}_{1} \mathfrak{p}_{2}\right) I D(Q)+\frac{1}{\mathfrak{p}_{3}+2} M_{1}(Q)+2\left(\mathfrak{q}_{1}+\mathfrak{q}_{2}+\mathfrak{q}_{3}\right)+3 \mathfrak{p}_{1} \mathfrak{p}_{2}+\mathfrak{q}_{1} \mathfrak{p}_{3} \\
& \cdot\left(\frac{2\left(\mathfrak{p}_{3}+2\right)+\mathfrak{q}_{1}}{\mathfrak{p}_{3}+1}\right)+\left(\mathfrak{p}_{1}+\mathfrak{q}_{1}\right)\left(\mathfrak{p}_{3}+2\right)+\frac{2 \mathfrak{q}_{1}\left(\mathfrak{p}_{2}+\mathfrak{q}_{3}\right)}{\mathfrak{p}_{3}+2},
\end{aligned}
$$

where $\quad \Gamma(T)=\sum_{q \in V(T)}\left(\omega_{T}(q) / \mu_{T}(q)\right) \quad$ and $\quad \Gamma(Y)=\quad$ Proof. By using Lemma 1 in (8), we obtain $\sum_{q \in V(Y)}\left(\omega_{Y}(q) / \mu_{Y}(q)\right)$.

$$
\begin{aligned}
\operatorname{IRM}_{2}\left(Q^{S} \triangleright\left(T^{V} \cup Y^{E}\right)\right)= & \sum_{q \in V\left(Q^{S} \triangleright\left(T^{V} \cup Y^{E}\right)\right)}\left|\mu_{Q^{S} \triangleright\left(T^{V} \cup Y^{E}\right)}(q)-\eta_{Q^{S} \triangleright\left(T^{V} \cup Y^{E}\right)}(q)\right| \\
= & \sum_{q \in V(T)}\left|\mu_{T}(q)+\mathfrak{p}_{1}-\frac{\omega_{T}(q)+\mathfrak{p}_{1} \mu_{T}(q)+2 \mathfrak{q}_{1}+\mathfrak{p}_{1} \mathfrak{p}_{2} \mid}{\mu_{T}(q)+\mathfrak{p}_{1}}\right| \\
& +\sum_{q \in V(Y)}\left|\mu_{Y}(q)+\mathfrak{q}_{1}-\frac{\omega_{Y}(q)+\mathfrak{q}_{1} \mu_{Y}(q)+\mathfrak{q}_{1}\left(\mathfrak{p}_{3}+2\right)}{\mu_{Y}(q)+\mathfrak{q}_{1}}\right| \\
& +\sum_{q \in V(Q)}\left|\mu_{Q}(q)+\mathfrak{p}_{2}-\frac{\mu_{Q}(q)\left(\mathfrak{p}_{3}+2\right)+2 \mathfrak{q}_{2}+\mathfrak{p}_{1} \mathfrak{p}_{2}}{\mu_{Q}(q)+\mathfrak{p}_{2}}\right| \\
& +\sum_{q=q q^{\prime} \in E(Q)}\left|\mathfrak{p}_{3}+2-\frac{\mu_{Q}(q)+\mu_{Q}\left(q^{\prime}\right)+2 \mathfrak{p}_{2}+2 \mathfrak{q}_{3}+\mathfrak{q}_{1} \mathfrak{p}_{3} \mid}{\mathfrak{p}_{3}+2}\right|
\end{aligned}
$$


Since for any real numbers $a$ and $b$, we have $|a-b| \leq a+b$. Therefore,

$$
\begin{aligned}
\operatorname{IRM}_{2}\left(Q^{S} \triangleright\left(T^{V} \cup Y^{E}\right)\right) \leq & \sum_{q \in V(T)}\left(\mu_{T}(q)+\mathfrak{p}_{1}\right)+\sum_{q \in V(Y)}\left(\mu_{Y}(q)+\mathfrak{q}_{1}\right)+\sum_{q \in V(Q)}\left(\mu_{Q}(q)+\mathfrak{p}_{2}\right) \\
& +\sum_{q=q q^{\prime} \in E(Q)}\left(\mathfrak{p}_{3}+2\right)+\sum_{q \in V(T)} \frac{\omega_{T}(q)+\mathfrak{p}_{1} \mu_{T}(q)+2 \mathfrak{q}_{1}+\mathfrak{p}_{1} \mathfrak{p}_{2}}{\mu_{T}(q)+\mathfrak{p}_{1}} \\
& +\sum_{q \in V(Y)} \frac{\omega_{Y}(q)+\mathfrak{q}_{1} \mu_{Y}(q)+\mathfrak{q}_{1}\left(\mathfrak{p}_{3}+2\right)}{\mu_{Y}(q)+\mathfrak{q}_{1}}+\sum_{q \in V(Q)} \frac{\mu_{Q}(q)\left(\mathfrak{p}_{3}+2\right)+2 \mathfrak{q}_{2}+\mathfrak{p}_{1} \mathfrak{p}_{2}}{\mu_{Q}(q)+\mathfrak{p}_{2}} \\
& +\sum_{q=q q^{\prime \prime} \in E(Q)} \frac{\mu_{Q}(q)+\mu_{Q}\left(q^{\prime}\right)+2 \mathfrak{p}_{2}+2 \mathfrak{q}_{3}+\mathfrak{q}_{1} \mathfrak{p}_{3}}{\mathfrak{p}_{3}+2} .
\end{aligned}
$$

Since $\quad\left(1 / \mu_{T}(q)+\mathfrak{p}_{1}\right) \leq\left(1 / \mu_{T}(q)\right), \quad\left(1 / \mu_{Y}(q)+\mathfrak{q}_{1}\right) \leq$ $\left(1 / \mu_{Y}(q)\right)$ and $\left(1 / \mu_{Q}(q)+\mathfrak{p}_{2}\right) \leq\left(1 / \mu_{Q}(q)\right)$ :

$$
\begin{aligned}
\operatorname{IRM}_{2}\left(Q^{S} \triangleright\left(T^{V} \cup Y^{E}\right)\right) \leq & \sum_{q \in V(T)}\left(\mu_{T}(Q)+\mathfrak{p}_{1}\right)+\sum_{q \in V(Y)}\left(\mu_{Y}(Q)+\mathfrak{q}_{1}\right)+\sum_{q \in V(Q)}\left(\mu_{Q}(Q)+\mathfrak{p}_{2}\right) \\
& +\sum_{q=q q^{\prime} \in E(Q)}\left(\mathfrak{p}_{3}+2\right)+\sum_{q \in V(T)}\left(\frac{\omega_{T}(Q)}{\mu_{T}(Q)}+\mathfrak{p}_{1}+\left(2 \mathfrak{q}_{1}+\mathfrak{p}_{1} \mathfrak{p}_{2}\right) \frac{1}{\mu_{T}(Q)}\right) \\
& +\sum_{q \in V(Y)}\left(\frac{\omega_{Y}(Q)}{\mu_{Y}(Q)}+\mathfrak{q}_{1}+\mathfrak{q}_{1}\left(\mathfrak{p}_{3}+2\right) \frac{1}{\mu_{Y}(Q)}\right) \\
& +\sum_{q \in V(Q)}\left(\left(\mathfrak{p}_{3}+2\right)+\left(2 \mathfrak{q}_{2}+\mathfrak{p}_{1} \mathfrak{p}_{2}\right) \frac{1}{\mu_{Q}(Q)}\right) \\
& +\frac{1}{\mathfrak{p}_{3}+2} \sum_{q_{=}=q q^{\prime} \in E(Q)}\left(\mu_{Q}(Q)+\mu_{Q}\left(q^{\prime}\right)+2 \mathfrak{p}_{2}+2 \mathfrak{q}_{3}+\mathfrak{q}_{1} \mathfrak{p}_{3}\right) \\
= & 2 \mathfrak{q}_{2}+\mathfrak{p}_{1} \mathfrak{p}_{2}+2 \mathfrak{q}_{3}+\mathfrak{q}_{1} \mathfrak{p}_{3}+2 \mathfrak{q}_{1}+\mathfrak{p}_{1} \mathfrak{p}_{2}+\mathfrak{q}_{1}\left(\mathfrak{p}_{3}+2\right)+\Gamma(T)+\mathfrak{p}_{1} \mathfrak{p}_{2}+\left(2 \mathfrak{q}_{1}+\mathfrak{p}_{1} \mathfrak{p}_{2}\right) I D(T) \\
& +\Gamma(Y)+\mathfrak{q}_{1} \mathfrak{p}_{3}+\mathfrak{q}_{1}\left(\mathfrak{p}_{3}+2\right) I D(Y)+\mathfrak{p}_{1}\left(\mathfrak{p}_{3}+2\right)+\left(2 \mathfrak{q}_{2}+\mathfrak{p}_{1} \mathfrak{p}_{2}\right) I D(Q)+\frac{1}{\mathfrak{p}_{3}+2} M_{1}(Q) \\
& +\frac{1}{\mathfrak{p}_{3}+2} M_{1}(Q)+2\left(\mathfrak{q}_{1}+\mathfrak{q}_{2}+\mathfrak{q}_{3}\right)+3 \mathfrak{p}_{1} \mathfrak{p}_{2}+\mathfrak{q}_{1} \mathfrak{p}_{3}\left(2+\frac{\mathfrak{q}_{1}}{\mathfrak{p}_{3}+2}\left(2 \mathfrak{p}_{2}+2 \mathfrak{q}_{3}+\mathfrak{q}_{1} \mathfrak{p}_{3}\right)+\left(\mathfrak{p}_{1}+\mathfrak{q}_{1}\right)\left(\mathfrak{p}_{3}+2\right)+\frac{2 \mathfrak{q}_{1}\left(\mathfrak{p}_{2}+\mathfrak{q}_{3}\right)}{\mathfrak{p}_{3}+2}\right. \\
= & \Gamma(T)+\Gamma(Y)+\left(2 \mathfrak{q}_{1}+\mathfrak{p}_{1} \mathfrak{p}_{2}\right) I D(T)+\mathfrak{q}_{1}\left(\mathfrak{p}_{3}+2\right) I D(Y)+\left(2 \mathfrak{q}_{2}+\mathfrak{p}_{1} \mathfrak{p}_{2}\right) I D(Q) \\
&
\end{aligned}
$$


The required result follows after some simplifications.

\section{Conclusions}

It is important to emphasis that as a large number of graphs are composed by smaller ones using graph products (and, as an outcome, their properties are strongly interconnected), the study of graph products is a timely and suitable research subject. Furthermore, the analysis of networks and graphs through their graph descriptors succeeds in playing an appreciable role to understand their underlying topologies, as it is commonly applied also in cheminformatics, bioinformatics, and biomedicine, where assessments derived through these invariants are valid for effectively communicating with the different hard tasks. Hence, the irregularity measures of connected graphs are manifested by a mathematical derivation approach. Our outcomes could perform a meaningful task in assessing the properties of underline structures.

\section{Data Availability}

No data are required to support the study.

\section{Conflicts of Interest}

The authors declare that they have no conflicts of interest.

\section{References}

[1] I. Gutman and N. Trinajstić, "Graph theory and molecular orbitals. Total $\varphi$-electron energy of alternant hydrocarbons," Chemical Physics Letters, vol. 17, no. 4, pp. 535-538, 1972.

[2] I. Gutman and O. E. Polansky, Mathematical Concepts in Organic Chemistry, Springer, New York, NY, USA, 1986.

[3] G. Rücker and C. Rücker, "On topological indices, boiling points, and cycloalkanes," Journal of Chemical Information and Computer Sciences, vol. 39, no. 5, pp. 788-802, 1999.

[4] L. B. Kier and L. H. Hall, Molecular Connectivity in Chemistry and Drug Research, Academic Press, New York, NY, USA, 1976.

[5] P. L. Krapivsky, S. Redner, and F. Leyvraz, "Connectivity of growing random networks," Physical Review Letters, vol. 85, no. 21, pp. 4629-4632, 2000.

[6] E. Estrada, "Atom-bond connectivity and the energetic of branched alkanes," Chemical Physics Letters, vol. 463, no. 4-6, pp. 422-425, 2008.

[7] E. Estrada, L. Torres, L. Rodríguez, and I. Gutman, "An atombond connectivity index: modeling the enthalpy of formation of alkanes," Indian Journal of Chemistry, vol. 37, pp. 849-855, 1998.

[8] M. Randic, "Characterization of molecular branching," Journal of the American Chemical Society, vol. 97, no. 23, pp. 6609-6615, 1975.

[9] H. Wiener, "Structural determination of paraffin boiling points," Journal of the American Chemical Society, vol. 69, no. 1, pp. 17-20, 1947.

[10] T. Reti, R. Sharfdini, A. Dregelyi-Kiss, and H. Hagobin, "Graph irregularity indices used as molecular discriptors in QSPR studies," MATCH Communications in Mathematical and in Computer Chemistry, vol. 79, pp. 509-524, 2018.

[11] E. Estrada, "Randic index, irregularity and complex biomolecular networks," Acta Chimica Slovenica, vol. 57, pp. 597603,2010 .
[12] S. Akhter, Z. Iqbal, A. Aslam, and W. Gao, "Mostar index of graph operations," 2020.

[13] F. K. Bell, "A note on the irregularity of graphs," Linear Algebra and its Applications, vol. 161, pp. 45-54, 1992.

[14] W. Gao, M. Aamir, Z. Iqbal, M. Ishaq, and A. Aslam, "On irregularity measures of some dendrimers structures," Mathematics, vol. 7, no. 3, p. 271, 2019.

[15] I. Gutman, "Irregularity of molecular graphs," Kragujevac Journal of Science, vol. 38, no. 38, pp. 71-81, 2016.

[16] Z. Iqbal, A. Aslam, M. Ishaq, and M. Aamir, "Characteristic study of irregularity measures of some nanotubes," Canadian Journal of Physics, vol. 97, no. 10, pp. 1125-1132, 2019.

[17] D. Zhao, Z. Iqbal, R. Irfan et al., "Comparison of irregularity indices of several dendrimers structures," Processes, vol. 7, no. 10, p. 662, 2019.

[18] L. V. Collatz and U. Sinogowitz, "Spektren endlicher grafen," in Abhandlungen Aus Dem Mathematischen Seminar Der Universitat Hamburg, vol. 21, pp. 63-77, Springer-Verlag, Hamburg, Germany, 1957.

[19] M. O. Albertson, "The irregularity of a graph," Ars Combinatoria, vol. 46, pp. 219-225, 1997.

[20] D. Dimitrov and T. Reti, "Graphs with equal irregularity indices," Acta Polytechnica Hungarica, vol. 11, pp. 41-57, 2014.

[21] W. Luo and B. Zhou, "On the irregularity of trees, unicyclic graphs with given matching number," Utilitas Mathematica, vol. 83, pp. 141-147, 2010.

[22] P. Hansen and H. Melot, "Variable neighborhood search for extremal graphs 9: bounding the irregularity of a graph," Discrete Mathematics \& Theoretical Computer Science, vol. 69, pp. 253-264, 2015.

[23] H. Abdo and D. Dimitrov, "The irregularity of graphs under graph operations," Discussiones Mathematicae Graph Theory, vol. 34, no. 2, pp. 263-278, 2014.

[24] H. Abdo, S. Brandt, and D. Dimitrov, "The total irregularity of a graph," Discrete Mathematics \& Theoretical Computer Science, vol. 16, pp. 201-206, 2014.

[25] D. Dimitrov and R. Skrekovski, "Comparing the irregularity, the total irregularity of graphs," Ars Mathematica Contemporanea, vol. 9, pp. 45-50, 2015.

[26] G. H. Fath-Tabar, "Old, new Zagreb indices of graphs," MATCH Communications in Mathematical and in Computer Chemistry, vol. 65, pp. 79-84, 2011.

[27] H. Abdo, N. Cohen, and D. Dimitrov, "Graphs with maximal irregularity," Filomat, vol. 28, no. 7, pp. 1315-1322, 2014

[28] R. Nasiri, H. R. Ellahi, A. Gholami, and G. H. Fath-Tabar, "The irregularity, total irregularity of Eulerian graphs, Iran," Journal of Mathematical Chemistry, vol. 9, pp. 101-111, 2018.

[29] I. Gutman, M. Togan, A. Yurttas, A. S. Cevik, and I. N. Cangul, "Inverse problem for sigma index," MATCH Communications in Mathematical and in Computer Chemistry, vol. 79, pp. 491-508, 2018.

[30] H. Abdo, D. Dimitrov, and I. Gutman, "Graphs with maximaloirregularity," Discrete Applied Mathematics, vol. 250, pp. 57-64, 2018.

[31] I. Gutman, "Stepwise irregular graphs," Applied Mathematics and Computation, vol. 325, pp. 234-238, 2018.

[32] B. Furtula and I. Gutman, "A forgotten topological index," Journal of Mathematical Chemistry, vol. 53, no. 4, pp. 11841190, 2015.

[33] A. Hamzeh and T. Reti, "An analogue of Zagreb index inequality obtained from graph irregularity measures," MATCH Communications in Mathematical and in Computer Chemistry, vol. 72, pp. 669-683, 2014. 
[34] S. Akhter, M. Imran, and Z. Raza, "Bounds for the general sum-connectivity index of composite graphs," Journal of Inequalities and Applications, vol. 2017, no. 1, 76 pages, 2017.

[35] A. R. Ashrafi, T. Došlić, and A. Hamzeh, "The Zagreb coindices of graph operations," Discrete Applied Mathematics, vol. 158, no. 15, pp. 1571-1578, 2010.

[36] M. Azari and A. Iranmanesh, "Computing the eccentricdistance sum for graph operations," Discrete Applied Mathematics, vol. 161, no. 18, pp. 2827-2840, 2013.

[37] N. De, S. M. A. Nayeem, and A. Pal, "The F-coindex of some graph operations," SpringerPlus, vol. 5, no. 1, p. 221, 2016.

[38] W. Gao, Z. Iqbal, Z. Iqbal, S. Akhter, M. Ishaq, and A. Aslam, "On irregularity descriptors of derived graphs," AIMS Mathematics, vol. 5, no. 5, pp. 4085-4107, 2020.

[39] M. Imran, S. Akhter, and Z. Iqbal, "Edge Mostar index of chemical structures and nanostructures using graph operations," International Journal of Quantum Chemistry, vol. 120, 2020.

[40] M. Imran, S. Akhter, and Z. Iqbal, "On the eccentric connectivity polynomial of $F$-sum of connected graphs," Complexity, vol. 2020, Article ID 5061682, 2020.

[41] Z. Iqbal, A. Aslam, M. Ishaq, and W. Gao, "The edge versions of degree-based topological descriptors of dendrimers," Journal of Cluster Science, vol. 31, no. 2, pp. 445-452, 2020.

[42] M. H. Khalifeh, H. Yousefi-Azari, and A. R. Ashrafi, "The first and second Zagreb indices of some graph operations," Discrete Applied Mathematics, vol. 157, no. 4, pp. 804-811, 2009.

[43] M. Berhe Belay and C. Wang, "The first general Zagreb coindex of graph operations," Applied Mathematics and Nonlinear Sciences, vol. 5, no. 2, pp. 109-120, 2020.

[44] S. Shirakol, M. Kalyanshetti, and S. M. Hosamani, "QSPR analysis of certain distance based topological indices," Applied Mathematics and Nonlinear Sciences, vol. 4, no. 2, pp. 371-386, 2019.

[45] M. Berhe and C. Wang, "Computation of certain topological coindices of graphene sheet and $\mathrm{C} 4 \mathrm{C} 8(\mathrm{~S})$ nanotubes and nanotorus," Applied Mathematics and Nonlinear Sciences, vol. 4, no. 2, pp. 455-468, 2019.

[46] S. Goyal, P. Garg, and V. N. Mishra, "New composition of graphs and their Wiener indices," Applied Mathematics and Nonlinear Sciences, vol. 4, no. 1, pp. 163-168, 2019.

[47] A. U. R. Virk, T. Abbas, and W. Khalid, "Multiplicative topological descriptors of Silicon carbide," Applied Mathematics and Nonlinear Sciences, vol. 4, no. 1, pp. 181-190, 2019.

[48] X. Zhang, H. M. Awais, M. Javaid, and M. K. Siddiqui, "Multiplicative Zagreb indices of molecular graphs," Journal of Chemistry, vol. 2019, Article ID 5294198, 19 pages, 2019.

[49] H. M. Awais, M. Jamal, and M. Javaid, "Topological properties of metal-organic frameworks," Main Group Metal Chemistry, vol. 43 , no. 1 , pp. $67-76,2020$.

[50] J.-B. Liu, M. Javaid, and H. M. Awais, "Computing Zagreb indices of the subdivision-related generalized operations of graphs," IEEE Access, vol. 7, pp. 105479-105488, 2019.

[51] F. Wen, Y. Zhang, and M. Li, "Spectra of subdivision vertexedge join of three graphs," Mathematics, vol. 7, no. 171, 2019.

[52] G. Indulal, "Spectrum of two new joins of graphs and infinite families of integral graphs," Kragujevac Journal of Mathematics, vol. 36, pp. 133-139, 2012. 